\title{
Acute Lymphocytic Meningoradiculoneuritis Complicating Primary Sjögren Syndrome
}

\author{
Salem Bouomrani ${ }^{1,2 *}$ and Mbarka Hadded ${ }^{1,2}$ \\ ${ }^{1}$ Department of Internal Medicine, Military Hospital of Gabes, Tunisia \\ ${ }^{2}$ Sfax Faculty of Medicine, University of Sfax, Tunisi
}

*Corresponding author: Salem Bouomrani, Department of Internal medicine,

Military Hospital of Gabes, Gabes 6000, Tunisia.

Received Date: June 22,2020

Published Date: July 06, 2020

\begin{abstract}
s
Acute lymphocytic meningoradiculoneuritis is an exceptional and unusual manifestation of neurological involvement in primary Sjögren syndrome (pSS). It may be the first manifestation revealing pSS and is often associated with greater disease activity. It represents a real diagnostic and therapeutic challenge for clinicians (specific pSS manifestation, infectious complication, or lymphomatous transformation of the disease?). We report the original observation of isolated acute lymphocytic meningoradiculoneuritis as the only manifestation of neuro-Sjögren in a 44-year-old Tunisian woman. As rare as it is, this clinical presentation of neuro-Sjögren deserves to be known by clinicians to avoid delayed diagnosis

Key words: Acute meningoradiculoneuritis; Sjögren syndrome; Lymphocytic meningitis; Polyradiculopathy; Neuro-Sjögren
\end{abstract}

\section{Introduction}

Sjögren syndrome (SS), also known as Gougerot-Sjögren syndrome, is an autoimmune connective tissue disease characterized by lymphocytic infiltration predominant in exocrine glands, and the production of autoantibodies (anti-nuclear, antiSSA, and anti-SSB antibodies) [1,2]. It can be primary (primary Sjögren's syndrome (pSS)) or secondary (secondary SS) if associated with other autoimmune diseases [1-3]. It is the most common connective tissue disease with an estimated prevalence of 5\% and an incidence at 4 per 1000 inhabitants per year [13]. The pathophysiology of this disease is not fully elucidated. It appears to be multifactorial including genetic predisposition, environmental, infectious, and hormonal factors [1,3]. Common features of pSS are xeropthalmia, xerostomia, pharynx, larynx, and vaginal dryness defining the classical sicca syndrome [1,2]. In approximately $10-15 \%$ of cases, lymphocytic infiltration can spread outside of the secretory glands causing various degrees of systemic (extraglandular) manifestations: pulmonary, renal, articular, digestive, neurological, cardiovascular, hematological, and cutaneous $[3,4]$. Neurological involvement is one of the most common extraglandular manifestations of pSS, and is by far dominated by peripheral neuropathies [5]. Meningoradiculoneuritis remains however exceptional and unusual during this disease [6]. We report the original observation of isolated lymphocytic meningoradiculoneuritis as the only manifestation of neuroSjögren in a 44-year-old Tunisian woman.

\section{Case Report}

A 44-year-old Tunisian woman, followed for four years for pSS, was admitted to our department for flaccid paraplegia of rapidly progressive installation evolving over the past two weeks. The diagnosis of pSS was based on the combination of: xerostomia, xerophthalmia, non-destructive non-deforming chronic polyarthritis, positive Schirmer's test, Chisholm stage III chronic lymphocytic sialadenitis on the biopsy of the minor salivary glands, and positive anti-nuclear, and anti-SSA autoantibodies). Subsequent 
investigations did not reveal any extra-glandular systemic damage (pulmonary, renal, neurological, digestive, hematological, skin, and cardiovascular). She was treated with hydroxychloroquine ( $400 \mathrm{mg} /$ day), prednisone $(10 \mathrm{mg} /$ day) and symptomatic treatment for mouth and eye sicca syndrome, with a favorable evolution. Systemic corticosteroid therapy was stopped after six months and the patient was kept only on hydroxychloroquine $200 \mathrm{mg} / \mathrm{d}$ and symptomatic treatment of sicca syndrome. The somatic examination noted a distal and proximal muscle deficit in the two lower limbs. Osteotendinous reflexes were abolished in the lower limbs, without amyotrophy or sensory level. The patient was conscious, oriented, without fever or cognitive impairment. The rest of the somatic examination was without abnormalities; in particular, no lymphadenopathy, organomegaly, palpable masses, purpura, or cranial neuropathy have been noted.

Basic biology showed a very marked inflammatory syndrome with an erythrocyte sedimentation rate (ESR) at $135 \mathrm{~mm} / \mathrm{H} 1$, a C-reactive protein at $18 \mathrm{mg} / \mathrm{l}$, and polyclonal hypergammaglobulinemia at $32 \mathrm{~g} / \mathrm{l}$. Procalcitonin was negative. The rest of the basic biological tests were within normallimits: total blood count, glycemia, calcemia, creatinine, plasma ionogram (sodium and potassium), transaminases, muscle enzymes, uric acid, and urinalysis. Magnetic resonance imaging (MRI) of the cervical spine concluded in a simple C1-C2 diastasis without spinal cord injury, which does not explain the patient's symptoms. The dorsolumbar spinal MRI was also normal. The lumbar puncture showed a clear and normotensive cerebrospinal fluid (CSF). Biochemical and cytological analysis of the CSF showed hyperproteinorrachia at $1.2 \mathrm{~g} / \mathrm{l}$, lymphocytic pleocytosis (220 leukocytes/mm3, 95\% lymphocytes), and normal glucorrachia. There were neither germs nor neoplastic cells. The CSF cultures were also negative. The electromyogram (EMG) concluded to polyradiculoneuropathy of the lower limbs, without myogenic damage.

Thus, the clinical presentation was that of acute aseptic lymphocytic meningoradiculoneuritis. Subsequent investigations have eliminated infectious and neoplastic causes, especially tuberculosis, borreliosis, herpes-zoster infection, and cerebromeningeal lymphoma. At the end of these explorations, the diagnosis retained was that of an aseptic (inflammatory) acute lymphocytic meningoradiculoneuritis complicating pSS. The patient was treated with systemic glucosteroids (3 boli of methylprednisolone at a dose of $1 \mathrm{~g} / \mathrm{d}$ for three days relayed by prednisone per os at a dose of $1 \mathrm{mg} / \mathrm{kg} / \mathrm{d}$ for one month, and followed by a gradual decrease).

The evolution was quickly favorable with possible standing and walking with assistance after ten days of treatment, normalization of the ESR and the serum proteins electrophoresis at three months, complete disappearance of the clinical neurological signs at two months of the treatment, and normalization of the CSF at six months of treatment.

\section{Discussion}

Neurological involvement during pSS was first described by Kaltreider \& Talal in 1969 [7]. It is currently considered among the most frequent extra-glandular manifestations of this disease, with a prevalence variously estimated according to the series at 8.5$70 \%$ [5,8-10]. Neurological damage in pSS (neuro-Sjögren) mainly affects the peripheral nervous system; involvement of the central nervous system (brain and spine) is much rarer [11,12]. According to the methods used for the detection of neuropathy, the frequency of peripheral neuropathy in pSS is estimated at $2-60 \%[5,6,10,11]$. The spectrum of peripheral neuropathies reported in pSS is very polymorphic: distal sensory polyneuropathy, axonal sensorimotor polyneuropathy, sensory ataxic neuropathy, mononeuritis multiplex, cranial neuropathy, chronic inflammatory demyelinating polyneuropathy, pure sensory neuropathy (small fiber neuropathy), truncal neuropathy, and sensorimotor polyradiculoneuropathy (Guillain-Barré syndrome) [5,8,13,14]. Polyradiculoneuropathy is exceptional during neuro-Sjören; indeed it was diagnosed in only $0.3 \%$ of cases in the French ASSESS cohort of 392 patients with pSS [6].

Aseptic meningitis is also rare in pSS [3-5]. Only one patient in the French ASSESS cohort of 392 patients with pSS, including 74 with neurological involvement, had meningitis $0.25 \%$ of pSS and $1.35 \%$ of neuro-Sjögren) [6]. Minor meningeal reaction (slight pleocytosis) is however more frequent; it was found in 9\% of patients followed for pSS with peripheral neuropathy in Pars $\mathrm{K}$ et al series [11]. The pathophysiology of peripheral neuropathy during pSS is not well known; several mechanisms may be involved: vasanervorum vasculitis, cryoglobulinemia, hypergammaglobulinemia, autoimmunity, and inflammation $[5,6,15]$. The main differential diagnoses to be discussed in front of acute meningoradiculoneuritis in patient with pSS are infectious causes (particularly Lyme disease/neuroborreliosis and Varicella-Zoster Virus reactivation) [16,17], and lymphomas (neurolymphomatosis) [18]. Opportunistic infections as well as lymphomatous transformation are common during pSS.

Treatment of pSS-associated peripheral neuropathy remains poorly codified; it includes systemic corticosteroids, immunosuppressants, intravenous gammaglobulin, plasma exchange, and recently biological agents (anti-tumor necrosis factor and monoclonal antibodies) [5,6,8,14,15,19]. Evolution remains unpredictable even under treatment, and frequent failures are observed, in particular in sensitive forms $[5,6,8,15]$.

\section{Conclusion}

Acute lymphocytic meningoradiculoneuritis remains an exceptional and unusual manifestation of neuro-Sjögren. It deserves to be known by clinicians to avoid delayed diagnosis. Early diagnosis and appropriate management are the only guarantees of a better prognosis, especially since this neuropathy may be the first 
manifestation revealing pSS and is often associated with greater disease activity.

\section{Acknowledgement}

None.

\section{Conflicts of Interest}

No conflict of interest.

\section{References}

1. Nair JJ, Singh TP (2017) Sjogren's syndrome: Review of the aetiology, Pathophysiology \& Potential therapeutic interventions. J Clin Exp Dent 9(4): e584-e589.

2. Stefanski AL, Tomiak C, Pleyer U, Dietrich T, Burmester GR, et al. (2017) The Diagnosis and Treatment of Sjögren's Syndrome. Dtsch Arztebl Int 114(20): 354-361.

3. Argyropoulou OD, Tzioufas AG (2018) Update on Sjögren's Syndrome 2018. Mediterr J Rheumatol 29(4): 193-198.

4. Ienopoli S, Carsons SE (2014) Extraglandular manifestations of primary Sjögren's syndrome. Oral Maxillofac Surg Clin North Am 26(1): 91-99.

5. Perzyńska-Mazan J, Maślińska M, Gasik R (2018) Neurological manifestations of primary Sjögren's syndrome. Reumatologia 56(2): 99105.

6. Carvajal Alegria G, Guellec D, Mariette X, Gottenberg JE, Dernis E, et al. (2016) Epidemiology of neurological manifestations in Sjögren's syndrome: data from the French ASSESS Cohort. RMD Open 2(1): e000179.

7. Kaltreider HB, Talal N (1969) The neuropathy of Sjögren's syndrome. Trigeminal nerve involvement. Ann Intern Med 70(4): 751-762.

8. Rafai MA, Boulaajaj FZ, Moutawakil F, Addali N, El Moutawakkil B, et al. (2009) Neurological manifestations revealing primitive GougerotSjogren syndrome: 9 cases. Joint Bone Spine 76(2): 139-145.

9. Teixeira F, Moreira I, Silva AM, Vasconcelos C, Farinha F, et al. (2013) Neurological involvement in Primary Sjögren Syndrome. Acta Reumatol Port 38(1): 29-36.
10. Tobón GJ, Pers JO, Devauchelle-Pensec V, Youinou P (2012) Neurological Disorders in Primary Sjögren's Syndrome. Autoimmune Dis 2012: 645967.

11. Pars K, Pul R, Schwenkenbecher P, Sühs KW, Wurster U, et al. (2017) Cerebrospinal Fluid Findings in Neurological Diseases Associated with Sjögren's Syndrome. Eur Neurol 77(1-2): 91-102.

12. Posso-Osorio I, Naranjo-Escobar J, Loaiza DM, Polo M, Echeverri A, et al. (2019) Neurological Involvement as the Initial Manifestation in Primary Sjögren's Syndrome - A Case Report. Curr Rheumatol Rev 15(3): 254258.

13. Yadav R, Krishnan P, Kulkarni GB, Yasha TC, Kumar MV, et al. (2011) Spectrum of Neuro-Sjogren's syndrome in a tertiary care center in south India. Ann Indian Acad Neurol 14(2): 111-115.

14. Yamashita H, Eri T, Ueda Y, Ozaki T, Takahashi H, et al. (2013) Diagnosis and treatment of primary Sjögren syndrome-associated peripheral neuropathy: a six-case series. Mod Rheumatol 23(5): 925-933.

15. Mekinian A, Ravaud P, Hatron PY, Larroche C, Leone J, et al. (2012) Efficacy of rituximab in primary Sjogren's syndrome with peripheral nervous system involvement: results from the AIR registry. Ann Rheum Dis 71(1): 84-87.

16. Hansen K, Crone C, Kristoferitsch W (2013) Lyme neuroborreliosis. Handb Clin Neurol 115: 559-575.

17. Snoeck R, Gérard M, Sadzot-Delvaux C, Andrei G, Balzarini J, et al. (1994) Meningoradiculoneuritis due to acyclovir-resistant varicella zoster virus in an acquired immune deficiency syndrome patient. J Med Virol 42(4): 338-347.

18. Li V, Jaunmuktane Z, Cwynarski K, Carr A (2019) Diagnostic delay in a case of T-cell neurolymphomatosis. BMJ Case Rep 12(12): e232538.

19. Kampylafka EI, Alexopoulos H, Dalakas MC, Tzioufas AG (2016) Immunotherapies for Neurological Manifestations in the Context of Systemic Autoimmunity. Neurotherapeutics 13(1): 163-178. 